\title{
A novel p.A191D matrilin-3 variant in a Vietnamese family with multiple epiphyseal dysplasia: a case report
}

Thuong Thi Ho ${ }^{1 \dagger}$, Linh Huyen Tran ${ }^{1,2 \dagger}$, Lan Thu Hoang ${ }^{3,4}$, Phuong Kim Thi Doan ${ }^{3,4}$, Trang Thi Nguyen ${ }^{3,4}$, Trang Hong Nguyen ${ }^{1}$, Hoai Thu Tran ${ }^{1}$, Ha Hoang ${ }^{1}$, Ha Hoang Chu ${ }^{1,2}$ and Anh Lan Thi Luong $3,4^{*}$

\begin{abstract}
Background: Multiple epiphyseal dysplasia (MED) is a common skeletal dysplasia that is characterized by variable degrees of epiphyseal abnormality primarily involving the hip and knee joints. Mutations in a gene encoding matrilin-3 (MATN3) have been reported as disease causing of autosomal dominant MED. The current study identified a novel c.572 C > A variant (p.A191D) in exon 2 of MATN3 in a Vietnamese family with MED.

Case presentation: A standard clinical tests and radiological examination were performed in an 8-year-old Vietnamese girl patient. The clinical examination showed that patient height was under average, with bent lower limbs, limited mobility and dislocation of the joints at both knees. Radiological documentation revealed abnormal cartilage development at the epiphysis of the femur and patella. The patient has a varus deformity of the lower limbs. The patient was diagnosed with autosomal dominant MED using molecular testing in the order of the coding sequences and flanking sequences of five genes: COMP (exons 8-19), MATN3 (exon 2), COL9A2 (exon 3), COL9A3 (exon 3), COL9A1 (exon 8) by Sanger sequencing. A novel heterozygous missense variant (c.572 C> A, p.A191D) in MATN3 was identified in this family, which were not inherited from parents. The p.A191D was predicted and classified as a pathogenic variant. When the two predicted structures of the wild type and mutant matrilin-3 were compared, the p.A191D substitution caused conformational changes near the substitution site, resulting in deformity of the $\beta$-sheet of the single A domain of matrilin- 3 .
\end{abstract}

Conclusions: This is the first Vietnamese MED family attributed to p.A191D matrilin-3 variant, and our clinical, radiological and molecular data suggest that the novel de novo missense variant in MATN3 contributed to MED.

Keywords: Multiple epiphyseal dysplasia, MATN3, Vietnamese case, Heterozygous, de novo missense variant, P.A191D matrilin-3

\footnotetext{
* Correspondence: luongthilananh27@gmail.com

${ }^{\dagger}$ Thuong Thi Ho and Linh Huyen Tran contributed equally to this work.

${ }^{3}$ Department of Biology \& Medical Genetic, Hanoi Medical University, 1 Ton

That Tung, Dong Da, Ha Noi, Viet Nam

${ }^{4}$ Genetic Counseling Center, Hanoi Medical University Hospital, 1 Ton That

Tung, Dong Da, Ha Noi, Viet Nam

Full list of author information is available at the end of the article
}

C C The Author(s). 2020 Open Access This article is licensed under a Creative Commons Attribution 4.0 International License, which permits use, sharing, adaptation, distribution and reproduction in any medium or format, as long as you give appropriate credit to the original author(s) and the source, provide a link to the Creative Commons licence, and indicate if changes were made. The images or other third party material in this article are included in the article's Creative Commons licence, unless indicated otherwise in a credit line to the material. If material is not included in the article's Creative Commons licence and your intended use is not permitted by statutory regulation or exceeds the permitted use, you will need to obtain permission directly from the copyright holder. To view a copy of this licence, visit http://creativecommons.org/licenses/by/4.0/. The Creative Commons Public Domain Dedication waiver (http://creativecommons.org/publicdomain/zero/1.0/) applies to the data made available in this article, unless otherwise stated in a credit line to the data. 


\section{Background}

Multiple epiphyseal dysplasia (MED) is a skeletal dysplasia of varying severity and is characterized by variable degrees of epiphyseal abnormality primarily involving the hip and knee joints [1-3]. The prevalence of autosomal dominant MED is estimated to be at least 1 in 10,000 newborns [4]. To date, mutation in five different genes have been shown to cause autosomal dominant MED; the genes encoding matrilin-3 (MATN3), cartilage oligomeric matrix protein $(C O M P)$, and the alpha 1-3 chains of type IX collagen (COL9A1, COL9A2, COL9A3) [2]. The relative proportions of five genes contributed to MED are different depending on ethnicity. A study of Jackson and his colleagues in the European Skeletal Dysplasia Network found that MATN3 pathogenic variants accounted for $24 \%$, COMP for $66 \%$, COL9A2 for $8 \%$, and COL9A3 for 2\% [5]. Whereas, the proportion of autosomal dominant MED attributed to MATN3 pathogenic variants was $55 \%$, followed by that to COMP (43\%), and COL9A2 (2\%) reported in 55 Korean individuals [6].

MATN3 incorporates coding sequence that comprises eight exons [7]. To date, at least 20 different variants in the MATN3 have been shown to cause a mild form of MED [8]. All of these missense variants and the polymorphism are found in the single A-domain of matrilin-3, which is encoded by exon 2 of MATN3 [4]. Matrilin-3 is the third member of a family of four extracellular matrix (ECM) proteins [9]. It consists of a single A-domain, four EGF repeats, and a coiled-coil domain that facilitates oligomerization. It forms either homotetramers or heterotetramers with matrilin-1 $[1,10]$. Matrilin $-3 / 1$ isolated from cartilage has been shown to bind with high affinity to both type IX collagen and cartilage oligomeric matrix protein, are believed to act as adaptor proteins in the ECM $[1,11]$.

In the present study, we used molecular testing that was shortened by targeting various exons that regularly occur variants in five genes cause MED in the order. The current study assessed in the order of the coding sequences and flanking sequences of five genes: COMP (exons 8-19), MATN3 (exon 2), COL9A2 (exon 3), COL9A3 (exon 3), COL9A1 (exon 8) associated with MED by Sanger sequencing, and characterized the associated clinical features. We identified a novel p.A191D matrilin-3 variant associated with MED from a Vietnamese family. Genetic analysis revealed heterozygous for the novel de novo missense variant in MATN3. Our genetic studies and clinical evaluation suggest that the novel p.A191D matrilin-3 is a pathogenic variant contributed to MED.

\section{Case presentation}

\section{Patient information}

The patient was an 8-year-old girl of two healthy parents. She has short stature of $105 \mathrm{~cm}$ height and weight of 40 kg. She has normal intellectual abilities. Her physical examination showed that her height was under average, with bent lower limbs (genu varum), limited mobility and dislocation of the joints at both knees. Personal and family histories were reviewed for each member of the threegeneration Vietnamese family (Fig. 1a).

\section{Past medical history}

Back in her prenatal stage, at 22 week old, the sonogram revealed the shortness of the limbs. However, the family still decided to keep the baby. There was no family history of musculoskeletal problems. Her karyotype results were $46, \mathrm{XX}$, with no abnormalities detected at chromosomal level (data not shown).

As the patient learnt to walk, the bending of the legs became more pronounced. She was admitted to the hospital and was provided with a cast for her legs in 3 months. After removing the cast, her condition was not improved. At the age of 6 , the patient received a knee arthrodesis procedure.

\section{Radiological findings}

The patient has a varus deformity of the lower limbs. Her limb lengths were lower than average: her femur was $33 \mathrm{~cm}$ long, and her tibia was $30 \mathrm{~cm}$ (Fig. 1b, c).

Radiological documentation revealed abnormal development in the femur and patella as ossification of the epiphyseal growth plates occurred irregularly. Tibial metaphysis are deformed and flatten; the femoral heads were short and flat. No deformity was detected in her hands and feet (Fig. 1d).

\section{Molecular data}

The study was approved by the Institute of Biotechnology and Hanoi Medical University Hospital (Ha Noi, Vietnam), and informed consent was obtained from all family members prior to blood sample collection. All family members from a Vietnamese family were recruited at Hanoi Medical University Hospital (Ha Noi, Vietnam) between April and May 2019. Genomic DNA samples were then extracted from the peripheral blood leucocytes of all family members using the Qiagen QIAamp DNA Mini kit (Qiagen Inc., Valencia, CA, USA) according to manufacturer's protocol. DNA concentration and purity was measured using a NanoDrop ${ }^{\mathrm{mm}}$ ND-1000 spectrophotometer (Thermo Fisher Scientific, Inc., Waltham, MA, USA). Genomic DNA samples were preserved at $20^{\circ} \mathrm{C}$ prior to use.

All sequences of exons 8-19 (COMP), exon 2 (MATN3), intron 3-exon 3 (COL9A2), intron 2-exon 3 (COL9A3), intron 7-exon 8 (COL9A1) were amplified in family members using PCR with Taq DNA polymerase (Thermo Fisher Scientific, Inc.), and specific primers sequences for COMP, MATN3, COL9A2 designed by previous studies $[1,7,12]$, respectively. We designed new 


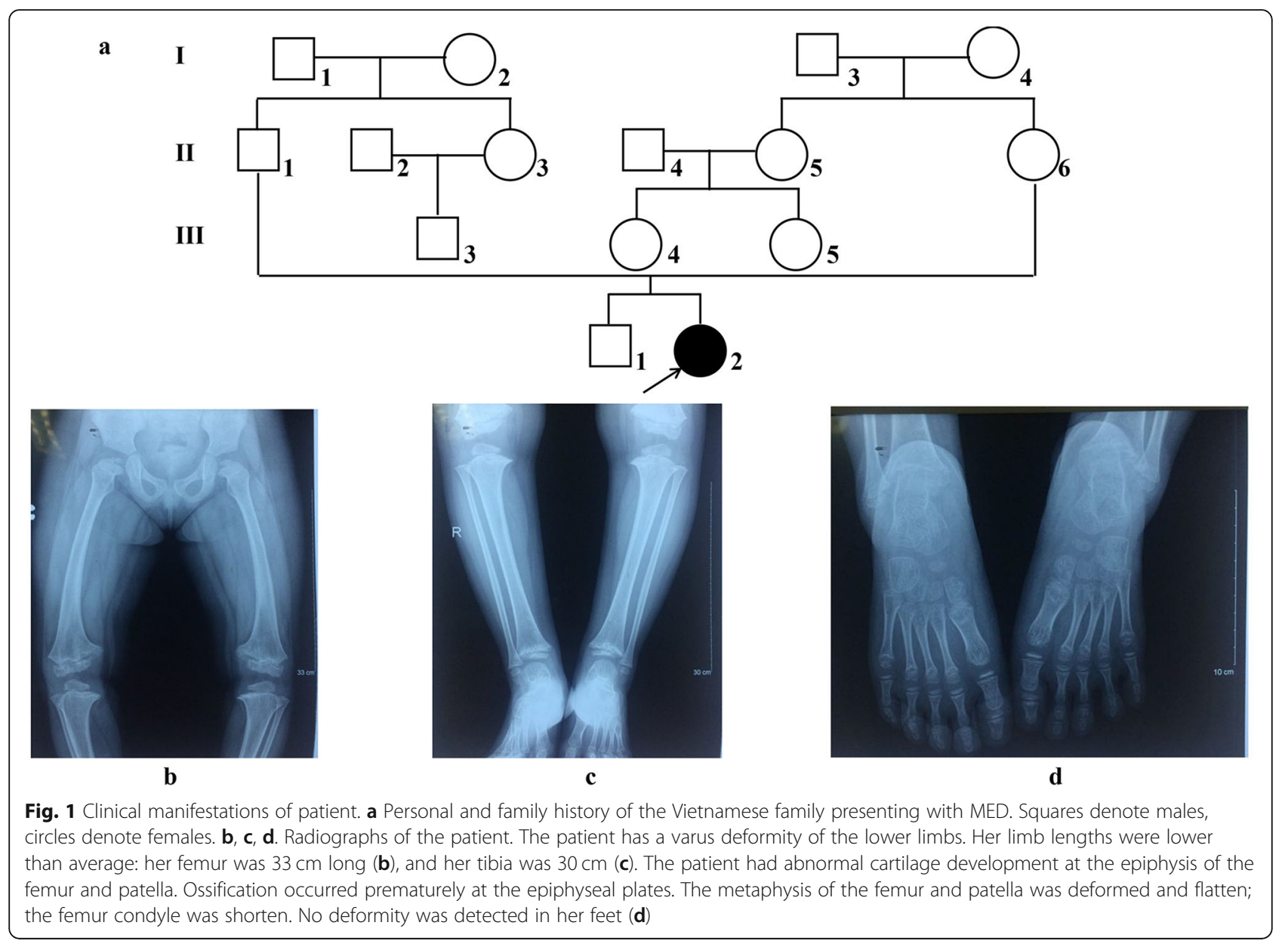

primers for amplification of target regions of $C O L 9 A 3$ and COL9A1. All primer sequences were listed in Supplementary 1 . DNA (100 ng) in a $50 \mu \mathrm{l}$ reaction was amplified. All reagents used for PCR were purchased from Thermo Fisher Scientific, Inc. The amplification included a single $5 \mathrm{~min}$ step at $94{ }^{\circ} \mathrm{C}$ followed by 40 cycles of $94{ }^{\circ} \mathrm{C}$ for $45 \mathrm{~s}$, annealing at temperatures listed (see Table 1) for $45 \mathrm{~s}$ and $72^{\circ} \mathrm{C}$ for $45 \mathrm{~s}$ followed by a final 10 min step at $72{ }^{\circ} \mathrm{C}$. Products were separated using $1 \%$ agarose gel electrophoresis and stained with ethidium bromide. DNA products then were purified using a QIAquick PCR Purification kit (Qiagen Inc.) and sequenced in each direction using an ABI3100 Genetic Analyzer (Thermo Fisher Scientific, Inc.). The variant detection was firstly performed for COMP and expanded to
MATN3, COL9A1, COL9A2, COL9A3 in order by Variant Reporter ${ }^{\text {Tix }}$ Software v2.0, Initial License (Thermo Fisher Scientific, Inc.), and analysed using SeqMan (version 2.3; Technelysium Pty, Ltd., South Brisbane, QLD, Australia), and compared against reference sequences obtained from NCBI with the code of NG_007070.1 (COMP), NG 008087.1 (MATN3), NC_000001.11 (COL9A2), NG_ 016353.1 (COL9A3), NG_011654.1 (COL9A1).

The Sanger sequencing revealed a de novo heterozygous variant at $\mathrm{cDNA}$ position c. $572 \mathrm{C}>\mathrm{A}$ in the exon 2 of the MATN3 in the patient, but not in unaffected parent and unaffected brother (Fig. 2a). This missense variant changes the codon for Alanine to Aspartic acid at the protein level (p.A191D). The heterozygous c. $572 \mathrm{C}>\mathrm{A}$ variant in exon 2 of MATN3 is evolutionarily

Table 1 Functional prediction of a novel de novo missense variant in MATN3

\begin{tabular}{|c|c|c|c|c|c|c|c|c|}
\hline Chr. & Position & Gene symbol & Transcript variant & Protein variant & $\begin{array}{l}\text { Mutation } \\
\text { Taster } \\
\text { score }\end{array}$ & Polyphen-2 & SIFT & Effect \\
\hline 2 & $20,205,723$ & MATN3 & $572 C>A$ & p.A191D & 0.999 & 1.000 & 0.02 & $\begin{array}{l}\text { - Damaging } \\
\text { - Disease causing (amino acid sequence changed) } \\
\text { - Protein features (might be) affected) } \\
\text { - Affect protein function }\end{array}$ \\
\hline
\end{tabular}






conserved among species (Fig. 2b). The following criteria were used to determine if the sequence variation was pathogenic variant: the chemical nature of the amino acids substituted, co-segregation with phenotype in family, interspecies amino acid conservation, absence in healthy individuals. Protein damaging structural information of the missense variant (c.572C > A, p.A191D) was predicted in MutationTaster [13], Polyphen-2 [14] and SIFT [15] to be 'Damaging' with a score of 0.999, 'Disease causing' with a score of 1.000 , and 'Affect protein function' with a score of 0.02 , respectively (Table 1 ). Based on the standards and guidelines for the interpretation of sequence variants of the American College of Medical Genetics and Genomics and the Association for Molecular Pathology [16], the c.572C > A (p.A191D) variant is classified to be 'Pathogenic'. In addition, the variant has not been found in any public database, including dbSNP (http://www.ncbi.nlm.nih.gov/projects/SNP/), 1000 genomes (http://www.1000genomes.org/), Human Gene Mutation Database (http://www.hgmd.cf.ac.uk/ac/index.php), Genome Aggregation Consortium (https://gnomad.broadinstitute.org/), Human Genetic Variation Database (http://www. hgvd.genome.med.kyoto-u.ac.jp/), ClinVar (https://www.ncbi. nlm.nih.gov/clinvar/), ESP6500 (http://evs.gs.washington.edu/ EVS/). Therefore, the de novo missense variant (c.572C $>\mathrm{A}$, p.A191D) was considered to be novel.

Moreover, two known coding-synonymous variants: c. $447 \mathrm{C}>\mathrm{T}$, c.615G $>\mathrm{A}$ in exon 2 of MATN3 were identified in patient and unaffected father, unaffected brother (Fig. 3). These variants are not conserved. The frequencies of these variants are above $40 \%$ in several public databases: 1000 Genomes, ExAC, GnomAD_ exomes and Vietnamese. Based on the standards and guidelines for the interpretation of sequence variants of the American College of Medical Genetics and Genomics and the Association for Molecular Pathology [16], these variants are classified to be 'Benign' for pathogenesis. All variants detected in this family, the variant frequencies in the population and variant classifications were shown in Table 2.

To understand the consequences of the Alanine to Aspartic acid substitution at amino acid position 191 in matrilin-3 protein, we used the model of the tertiary structure of human matrilin-3 (UniProt ID: O15232) constructed by SWISS-MODEL [17-21] as the wild-type (Fig. 4a) and introduced the p.A191D variant into this model using PyMOL v1.3 (Schrödinger, LLC, New York, USA) to create the mutantmatrilin-3 protein (Fig. 4b). When the two predicted structures of the wild type and the mutatedmatrilin- 3 were compared, the p.A191D substitution caused conformational changes near the mutation site, resulting in deformity of the $\beta$-sheet of the single A domain of matrilin- 3 .

\section{Discussion and conclusion}

In the current study, we shortened molecular testing of the patient by targeting various exons that regularly 
a
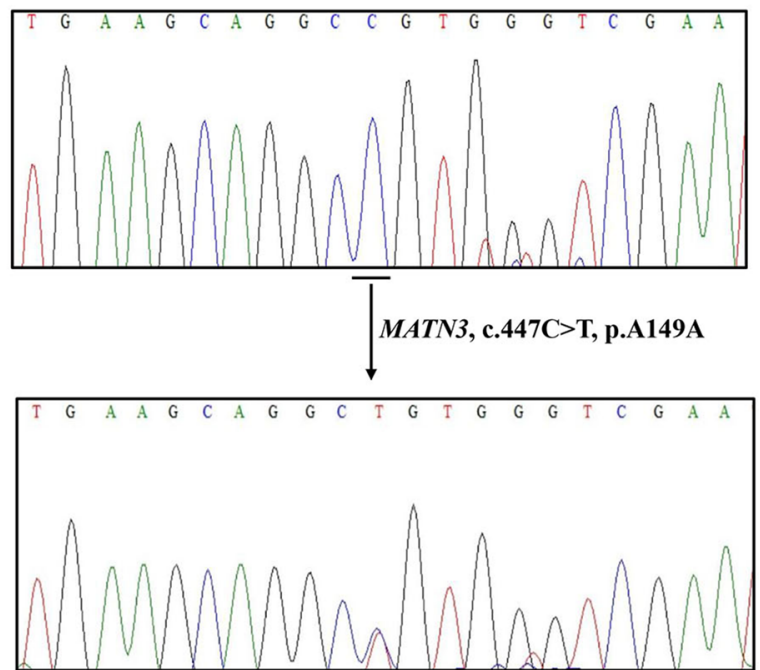

Fig. 3 Identification of two known coding-synonymous variants in MATN3. a The DNA-sequence electropherogram of c.447C > T coding-synonymous variant. $\mathbf{b}$ The DNA-sequence electropherogram of c.615G > A coding-synonymous variant b
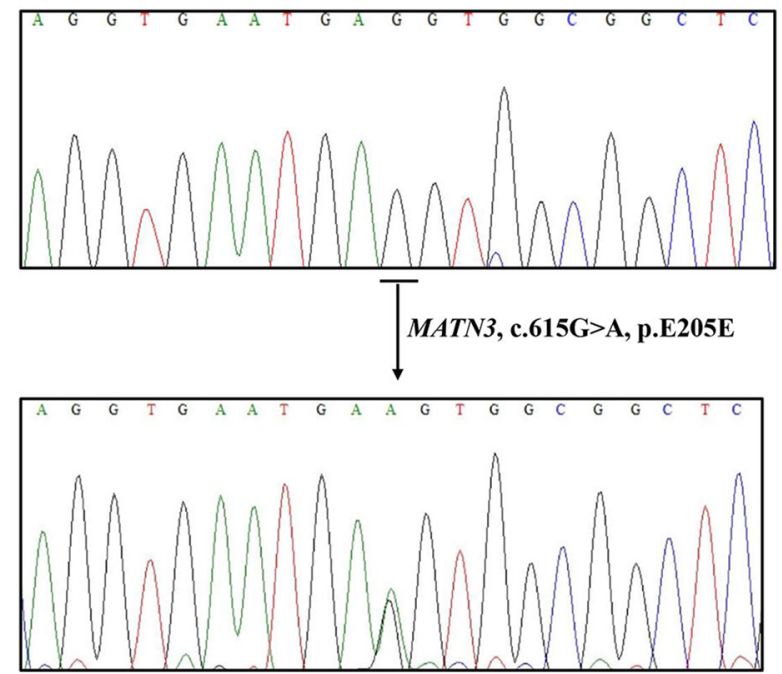

occur mutations in five genes cause MED in the order. We identified a novel de novo missense variant (c.572C > A, p.A191D) and two known coding-synonymous variants (c.447C > T, p. $(=)$; c. $615 \mathrm{G}>\mathrm{A}, \mathrm{p} .(=))$ in MATN3 in the patient. The novel c.572C > A, p.A191D variant was predicted by three predictors as 'Disease causing' with autosomal dominant MED, suggesting that it is pathogenic variant. Whereas two coding-synonymous variants (c.447C > T, p.(=); c.615G > A, p. (=)) with allele frequencies above $40 \%$ in the general population are classified as benign variants. These data therefore extend the range of MATN3 variants identified to date. Several missense variants in MATN3 have been previously reported to be associated with MED, including p.R121W, p.V194D [22], p.T120M, p.E134K, p.I192N, and p.A219D [23], p.T120M, p.R121W, p.L146R, p.G159G, p.I192T, p.R209P, p.V220A

Table 2 List of variants in MATN3 in family

\begin{tabular}{|c|c|c|}
\hline & Variant & Variant classification \\
\hline \multirow[t]{2}{*}{ Father II.1 } & $\begin{array}{l}\text { c. } 447 \mathrm{C}>\mathrm{T}, \\
\text { p. }(=)\end{array}$ & Benign \\
\hline & $\begin{array}{l}\text { c. } 615 G>A, \\
\text { p. }(=)\end{array}$ & Benign \\
\hline Mother II. 6 & - & \\
\hline \multirow[t]{2}{*}{ Brother III.1 } & c. $447 \mathrm{C}>\mathrm{T}, \mathrm{p} .(=)$ & Benign \\
\hline & c.615G > A, $p(=)$ & Benign \\
\hline \multirow[t]{3}{*}{ Patient III.2 } & c.447C > T, p. $(=)$ & Benign \\
\hline & c.615G > A, p. $(=)$ & Benign \\
\hline & c.572C > A, p.A191D & Pathogenic \\
\hline
\end{tabular}

(-): No variant was detected
[6]. All the MED variants in MATN3 are missense variants found in exon 2 [23].

The severity of pathogenic variants can modify the severity of MED signs and symptoms [4, 23, 24]. MED symptom resulting from MATN3 pathogenic variants is characterized by significant involvement in knee abnormalities that are similar to those in individuals with a COL9A2 pathogenic variant; the hip abnormalities are more severe (although not as severe as those in individuals with a COMP pathogenic variant) [4, 25]. However, more intra- and interfamilial variability is evident in MED caused by MATN3 pathogenic variants [4]. In this study, our patient carried p.A191D variant had under average height, with bent lower limbs, limited mobility and dislocation of the joints at both knees. Radiological analysis showed that she had abnormal development in the femur and patella because ossification of her epiphyseal growth plates occurred irregularly. Various missense variants in MATN3 that is near to the p.A191D variant in our patient were reported with diverse MED symptoms. Kim and colleagues identified a patient carrying a p.I192T variant located in $\beta$-sheet of the single $A$ domain of matrilin-3. The patient in that study was 8year-old boy who had MED symptom of gait abnormality, but he had normal height. Radiographic features of his hips indicated that there was a dense, crescent shape of the femoral heads with fragmentation on the left side mimics Legg-Calve-Perthes disease [6]. In addition, there was another p.V194D variant that is next to the mutation in our patient was identified by Chapman and his colleagues [22]. That patient presented at the age of 10 years with knee pain after exercise, genu valga and 


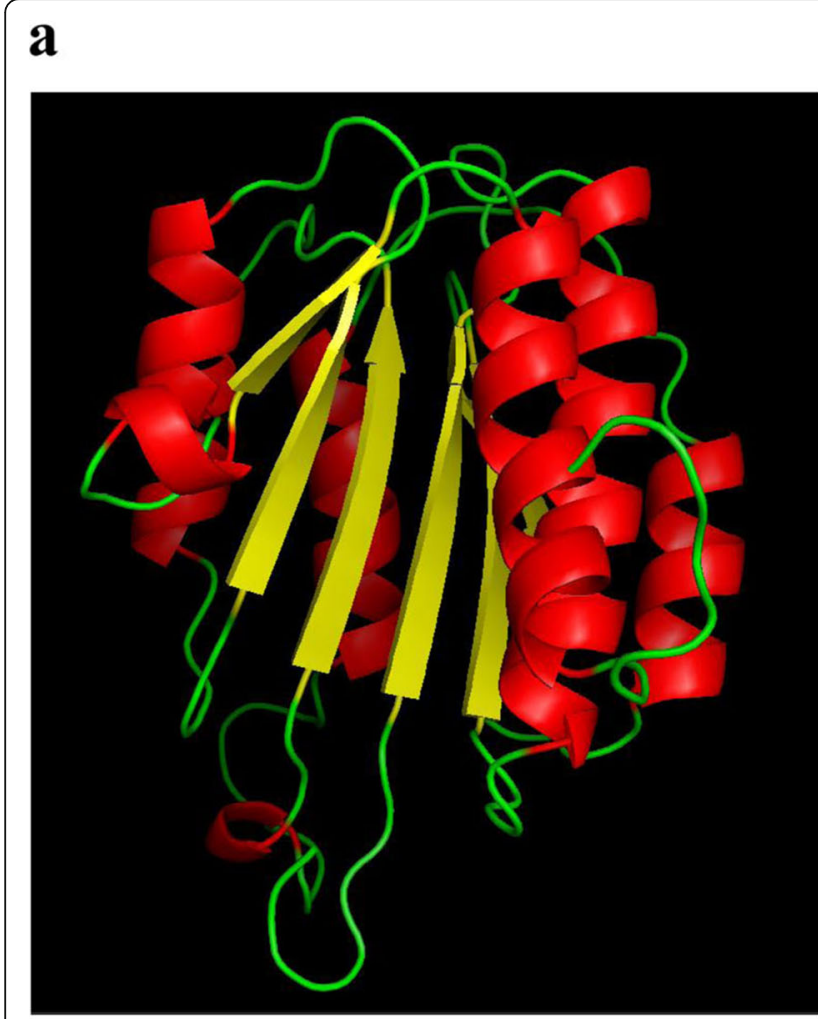

Wild type MATN3 protein b

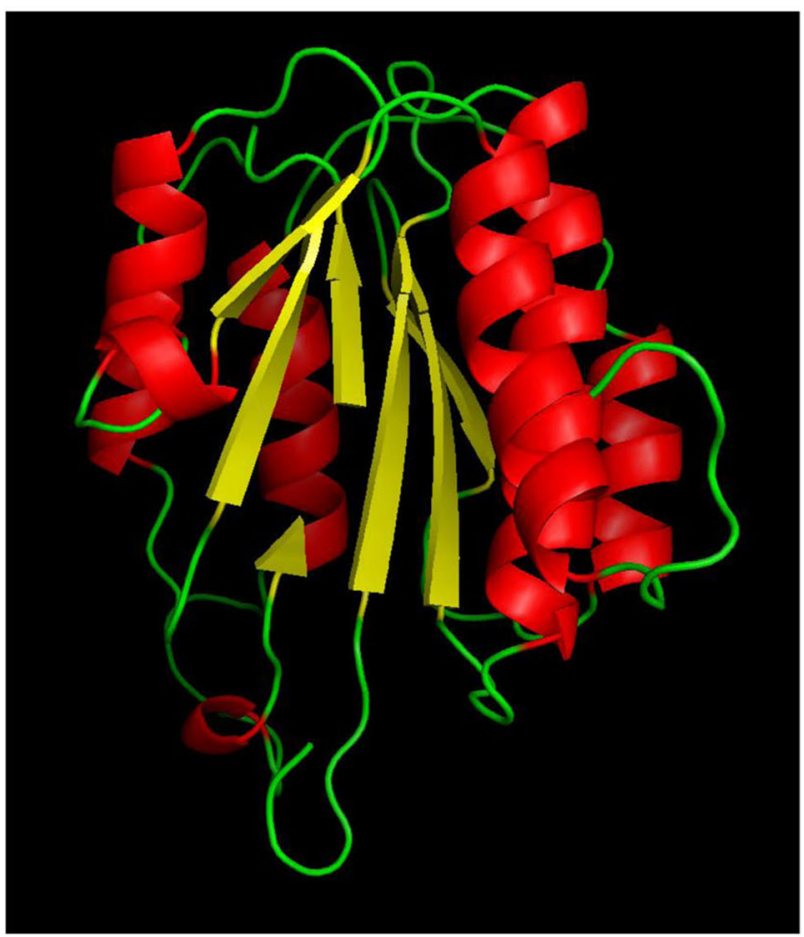

Mutant MATN3 protein

Fig. 4 Modeling studies of novel p.A191D variant in MATN3. a The model of the tertiary structure of human matrilin-3 (UniProt ID: O15232) as the wild type was constructed by SWISS-MODEL. $\mathbf{b}$ The model of the tertiary structure of mutated human matrilin-3was built by introducing the p.A191D variant into the wild type model using PyMOL v1.3. The p.A191D substitution caused conformational changes near to the variant site, resulting in losing a part of $\beta$-sheet of the single A domain of matrilin-3. $\beta$-sheet and a-helix structures of matrilin-3 were shown as yellow and red color, respectively

normal stature. Radiographs of his skeleton revealed that he had normal spines and hands but predominant involvement of the epiphyses of hips, knees and ankles [22]. Therefore, these supports previous observations that the severity of MED signs and symptoms can vary among affected patients due to severity of the pathogenic variants even with very close variants [23, 24].

The p.A191D variant in matrilin-3 is located within the central $\beta$-sheet of the single A domain of matrilin-3. The change of Alanine to Aspatic acid at position 191 caused conformational changes near to the substitution site, resulting in malformation of $\beta$-sheet of the single A domain of matrilin-3. The consequences of various missense variants in the $\beta$-strands of the A domain of matrilin-3 protein had been reported. The previous study demonstrated that various missense variants in the $\beta$-strands of the A domain of matrilin-3 protein delayed folding, prevented correct intra-molecular disulfide bond formation, and results in the retention of mutated matrilin-3 in the rough endoplasmic reticulum both in vitro $[1,26]$ and in vivo $[27,28]$. Leighton and his colleagues indicated that the expression of p.V194D pathogenic variant caused endoplasmic reticulum stress and an unfolded protein response in an MED mouse model [27]. In addition, matrilins have been found in collagen-dependent and collagen-independent filament networks within the tissues in which they are expressed and may perform analogous functions in these different tissues $[4,29,30]$. Moreover, matrilin-3 has been shown to interact with high affinity to both cartilage oligomeric matrix protein and type IX collagen through A domain, and act as adaptor proteins in the ECM [10, 29-31]. Therefore, the distortion of $\beta$-sheet of the single A domain of matrilin-3 caused by p.A191D = variant might affect to the adaptor protein function of matrilin-3, and might lead to low binding affinity to cartilage oligomeric matrix protein and type IX collagen protein.

In summary, a novel de novo missense variant c.572C > A (p.A191D) in exon 2 of the MATN3 with evolving clinical and radiological features of MED was identified in a Vietnamese family. These results may not only expand the reported variant spectrum of MATN3, 
but may also provide useful information to aid clinicians to confirm the diagnosis of MED. More genetic studies are required to determine the frequency of this variant and this diagnostic credibility.

\section{Supplementary information}

Supplementary information accompanies this paper at https://doi.org/10. 1186/s12891-020-03222-4.

Additional file 1. Primer sequences for amplification.

Additional file 2. The frequencies of two known variants in public database.

\section{Abbreviations}

MED: Multiple epiphyseal dysplasia; MATN3: Gene encoding matrilin-3; COMP: Gene encoding cartilage oligomeric matrix protein; COL9A1, COL9A2, COL9A3: Gene encoding the alpha 1-3 chains of type IX; ECM: Extracellular matrix; SNP: Single nucleotide polymorphism

\section{Acknowledgements}

The authors would like to thank the patient and family members for their participation.

\section{Authors' contributions}

ALTL, HHC, LHT and TTH designed radiological and molecular experiments, respectively. TTH, THN and LHT drafted manuscript; ALTL and LTH performed the clinical assessments of the patients; PKTD, TTN characterized the patients clinically; HTT and HH contributed to the Sanger sequencing; LHT, TTH contributed to the mutation analysis. THN and HHC performed protein structure modeling analysis. All authors read and approved the final manuscript.

\section{Funding}

The present study was financially supported by Hanoi Medical University Hospital (Viet Nam) and technically supported by the National Key Laboratory of Gene Technology, Institute of Biotechnology (Vietnam Academy of Science and Technology).

\section{Availability of data and materials}

The datasets used and/or analysed during the current study are available from the corresponding author on reasonable request.

\section{Ethics approval and consent to participate}

All procedures were performed in a bioassay laboratory and approved by the local ethics committee of Hanoi Medical University Hospital and Institute of biotechnology, Vietnam Academy of Science and Technology (Ha Noi, Viet Nam)

\section{Consent for publication}

The parent of the patient consented to the publication of the case and any accompanying images with written consent.

\section{Competing interests}

The authors declare that they have no competing interests.

\section{Author details}

${ }^{1}$ National Key Laboratory of Gene Technology, Institute of Biotechnology (IBT), Vietnam Academy of Science and Technology (VAST), 18 Hoang Quoc Viet, Cau Giay, Ha Noi, Viet Nam. ${ }^{2}$ Graduate University of Science and Technology, Vietnam Academy of Science and Technology, 18 Hoang Quoc Viet, Cau Giay, Ha Noi, Viet Nam. ${ }^{3}$ Department of Biology \& Medical Genetic, Hanoi Medical University, 1 Ton That Tung, Dong Da, Ha Noi, Viet Nam. ${ }^{4}$ Genetic Counseling Center, Hanoi Medical University Hospital, 1 Ton That Tung, Dong Da, Ha Noi, Viet Nam.
Received: 12 September 2019 Accepted: 18 March 2020

Published online: 07 April 2020

\section{References}

1. Cotterill SL, Jackson GC, Leighton MP, et al. Multiple epiphyseal dysplasia mutations in MATN3 cause misfolding of the A-domain and prevent secretion of mutant matrilin-3. Hum Mutat. 2005:26(6):557-65.

2. Briggs MD, Chapman KL. Pseudoachondroplasia and multiple epiphyseal dysplasia: Mutation review, molecular interactions, and genotype to phenotype correlations. Hum Mutat. 2002;19(5):465-78.

3. Unger $\mathrm{S}$, Hecht JT. Pseudoachondroplasia and multiple epiphyseal dysplasia: New etiologic developments. Am J Med Genet. 2001;106:244-50.

4. Briggs MD, Wright MJ, Mortier GR. Multiple Epiphyseal Dysplasia, Autosomal Dominant. 2003 [Updated 2019]. In: Adam MP, Ardinger HH, Pagon RA, et al., editors. GeneReviews ${ }^{\oplus}$. Seattle (WA): University of Washington, Seattle; 1993-2019. https://www.ncbi.nlm.nih.gov/books/NBK1123/.

5. Jackson GC, Mittaz-Crettol L, Taylor JA, Mortier GR, Spranger J, Zabel B, et al. Pseudoachondroplasia and multiple epiphyseal dysplasia: a 7-year comprehensive analysis of the known disease genes identify novel and recurrent mutations and provides an accurate assessment of their relative contribution. Hum Mutat. 2012;33:144-57.

6. Kim OH, Park H, Seong MW, Cho TJ, Nishimura G, Superti-Furga A, Unger S, et al. Revisit of multiple epiphyseal dysplasia: ethnic difference in genotypes and comparison of radiographic features linked to the COMP and MATN3 genes. Am J Med Genet A. 2011;155A:2669-80.

7. Belluoccio D, Schenker T, Baici A, Trueb B. Characteriza-tion of human matrilin-3 (MATN3). Genomics. 1998;53:391-4.

8. Bell PA, Piróg KA, Fresquet M, Thornton DJ, Boot-Handford RP, Briggs MD. Loss of matrilin 1 does not exacerbate the skeletal phenotype in a mouse model of multiple epiphyseal dysplasia caused by a Matn3 V194D mutation. Arthritis Rheum. 2012;64(5):1529-39.

9. Wagener R, Kobbe B, Paulsson M. Primary structure of matrilin-3, a new member of a family of extracellular matrix proteins related to cartilage matrix protein (matrilin-1) and von Willebrand factor. FEBS Lett. 1997;413: 129-34

10. Klatt AR, Nitsche DP, Kobbe B, Morgelin M, Paulsson M, Wagener R. Molecular structure and tissue distribution of matrilin-3, a filament-forming extracellular matrix protein expressed during skeletal development. J Biol Chem. 2000:275:3999-4006.

11. Wagener R, Ehlen HW, Ko YP, Kobbe B, Mann HH, Sengle G, Paulsson M. The matrilins-adaptor proteins in the extracellular matrix. FEBS Lett. 2005; 579:3323-9.

12. Muragaki $Y$, Mariman EC, van Beersum SE, Perala M, van Mourik JB, Warman $\mathrm{ML}$, Olsen BR, Hamel BC. A mutation in the gene encoding the alpha 2 chain of the fibril- associated collagen IX, COL9A2, causes multiple epiphyseal dysplasia (EDM2). Nat Genet. 1996;12:103-5.

13. Ramensky V, Bork P, Sunyaev S. Human non-synonymous SNPs: Server and survey. Nucleic Acids Res. 2002;30:3894-900.

14. Ng PC, Henikoff S. Predicting deleterious amino acid substitutions. Genome Res. 2001:11:863-74.

15. Schwarz JM, Cooper DN, Schuelke M, Seelow D. MutationTaster2: mutation prediction for the deep-sequencing age. Nat Methods. 2014;11(4):361-2.

16. Richards S, Aziz N, Bale S, Bick D, Das S, Gastier-Foster J, et al. Standards and guidelines for the interpretation of sequence variants: a joint consensus recommendation of the American College of Medical Genetics and Genomics and the Association for Molecular Pathology. Genet Med. 2015: 17(5):405-24.

17. Waterhouse A, Bertoni M, Bienert S, Studer G, Tauriello G, Gumienny R, Heer FT, de Beer TAP, Rempfer C, Bordoli L, Lepore R, Schwede T. SWISS-MODEL: homology modelling of protein structures and complexes. Nucleic Acids Res. 2018:46(W1):W296-303.

18. Bienert S, Waterhouse A, de Beer TAP, Tauriello G, Studer G, Bordoli L, Schwede T. The SWISS-MODEL Repository - new features and functionality. Nucleic Acids Res. 2017:45:D313-9.

19. Guex N, Peitsch MC, Schwede T. Automated comparative protein structure modeling with SWISS-MODEL and Swiss-PdbViewer: A historical perspective. Electrophoresis. 2009:30:5162-73.

20. Benkert P, Biasini M, Schwede T. Toward the estimation of the absolute quality of individual protein structure models. Bioinformatics. 2011;27: $343-50$ 
21. Bertoni M, Kiefer F, Biasini M, Bordoli L, Schwede T. Modeling protein quaternary structure of homo- and hetero-oligomers beyond binary interactions by homology. Sci Rep. 2017;7:10480.

22. Chapman KL, Mortier GR, Chapman K, Loughlin J, Grant ME, Briggs MD. Mutations in the region encoding the von Willebrand factor A domain of matrilin-3 are associated with multiple epiphyseal dysplasia. Nat Genet. 2001;28:393-6.

23. Jackson GC, Barker FS, Jakkula E, Czarny-Ratajczak M, Makitie O, Cole WG, et al. Missense mutations in the beta strands of the single A-domain of matrilin 3 result in multiple epiphyseal dyspla-sia. J Med Genet. 2004;41:52-9.

24. Mäkitie O, Mortier GR, Czarny-Ratajczak M, Wright MJ, Suri M, Rogala P, Freund M, Jackson GC, Jakkula E, Ala-Kokko L, Briggs MD, Cole WG. Clinical and radiographic findings in multiple epiphyseal dysplasia caused by MATN3 mutations: description of 12 patients. Am J Med Genet A. 2004; 125A:278-84.

25. Mortier GR, Chapman K, Leroy JL, Briggs MD. Clinical and radiographic features of multiple epiphyseal dysplasia not linked to the COMP or type IX collagen genes. Eur J Hum Genet. 2001;9:606-12.

26. Otten C, Wagener R, Paulsson M, Zaucke F. Matrilin-3 mutations that cause chondrodysplasias interfere with protein trafficking while a mutation associated with hand osteoarthritis does not. J Med Genet. 2005;42:774-9.

27. Leighton MP, Nundlall S, Starborg T, Meadows RS, Suleman F, Knowles L, Wagener R, Thornton DJ, Kadler KE, Boot-Handford RP, Briggs MD. Decreased chondrocyte proliferation and dysregulated apoptosis in the cartilage growth plate are key features of a murine model of epiphyseal dysplasia caused by a matn3 mutation. Hum Mol Genet. 2007:16:1728-41.

28. Nundlall S, Rajpar MH, Bell PA, Clowes C, Zeeff LA, Gardner B, Thornton DJ, Boot-Handford RP, Briggs MD. An unfolded protein response is the initial cellular response to the expression of mutant matrilin-3 in a mouse model of multiple epiphyseal dysplasia. Cell Stress Chaperones. 2010;15:835-49.

29. Mann HH, Ozbek S, Engel J, Paulsson M, Wagener R. Interactions between the cartilage oligomeric matrix protein and matrilins. Implications for matrix assembly and the pathogenesis of chondrodysplasias. J Biol Chem. 2004; 279:25294-8.

30. Fresquet M, Jowitt TA, Ylöstalo J, Coffey P, Meadows RS, Ala-Kokko L, Thornton DJ, Briggs MD. Structural and functional characterization of recombinant matrilin-3 A-domain and implications for human genetic bone diseases. J Biol Chem. 2007;282:34634-43.

31. Fresquet M, Jowitt TA, Stephen LA, Ylöstalo J, Briggs MD. Structural and functional investigations of Matrilin-1 A-domains reveal insights into their role in cartilage ECM assembly. J Biol Chem. 2010;285:34048-61.

\section{Publisher's Note}

Springer Nature remains neutral with regard to jurisdictional claims in published maps and institutional affiliations.

Ready to submit your research? Choose BMC and benefit from:

- fast, convenient online submission

- thorough peer review by experienced researchers in your field

- rapid publication on acceptance

- support for research data, including large and complex data types

- gold Open Access which fosters wider collaboration and increased citations

- maximum visibility for your research: over $100 \mathrm{M}$ website views per year

At $\mathrm{BMC}$, research is always in progress.

Learn more biomedcentral.com/submissions 